\title{
Hall voltage sign reversal in type II superconductors
}

\author{
Jan Koláček, Petr Vašek \\ Institute of Physics, ASCR, Cukrovarnická 10,16253 Prague 6, Czech Republic
}

\begin{abstract}
The Hall voltage sign reversal is consistently explained by the model in which vortices with the superconducting and normal state charge carriers are regarded as three subsystems mutually connected by interactions. The equations of motion for these three subsystems are solved simultaneously and a new formula for the Hall resistivity in the flux flow regime is obtained. It is shown that it is possible to explain qualitatively experimental data by this model.
\end{abstract}

Keywords: mixed state, Hall effect, electrical resistivity

\section{INTRODUCTION}

The dynamics of vortices shows a rich behaviour that is still not completely understood. There is a general belief that moving vortices significantly contribute to the Hall voltage sign reversal in the mixed state of superconductors which is one of the most intriguing and controversial transport phenomena. One of the earliest attempts to explain this Hall anomaly was made using Bardeen and Stephen [1] or Nozieres and Vinnen [2] models. However these theories are unable to provide a satisfactory explanation of experimental data.

Starting from the fact that vortices moving with velocity $\mathbf{v}_{L}$ create electric field $\mathbf{E}_{v}=-\mathbf{v}_{L} \times \mathbf{B}_{v}\left(\mathbf{B}_{v}\right.$ is magnetic field carried by vortex) Hagen at all [3] argued that vortices moving antiparallel to the transport current must be responsible for the Hall voltage sign reversal. Several theories were suggested to explain this behavior. They took into account particle hole asymmetry [4. a pinned vortex lattice [8]. Wang et al. [9] developed a theory explaining observed results by pinning and thermal fluctuation effects. Recently Zhu et al. 10] extended Wang's theory taking into account also vortex-vortex interaction. Effect of pinning on the vortex Hall resistivity was also dealt in 11. However, measurements made on samples with different correlated disorder 12] confirmed that sign reversal is an intrinsic property of type II superconductors and also other experiments did not confirm the role of pinning in Hall voltage change of sign [13,14. Also measurements at high current densities 15] suppressing the effective pinning show only little effect. Attention was devoted also to the attempt to explain the Hall anomaly by taking into account the charge of the vortex core 16, 17. As the chemical potential differs in the normal and the superconducting phases, the vortex core may become charged. Simple physical considerations show that the charge of the vortex core is opposite to the sign of the dominant charge carriers 17] and it was recently experimentally verified experimen- tally by Matsuda and Kumagai 18]. Feigelman's 16 formula can be used to describe the experimental data quite well (see e.g. 19,20]), but to achieve it the carrier density in the core must be supposed to be larger than outside, which seems to be incorrect. On the other hand Khomskii [17] supposes the correct sign of the vortex core charge, but Feigelman [16] argues that the additional transverse force used by Khomskii has the opposite sign, which contradicts the result for the Magnus force in the Galilean invariant case. Moreover Wang et.al. argue that in the low magnetic field limit both these models lead to H-dependence of Hall angle, which violates their experimental finding obtained in ultra low magnetic field [21]. Currently Hall anomaly is still considered to be an unsolved problem and new models are proposed (see e.g. the mixed charge model proposed by Ji and Wang [22]).

For correct understanding of the vortex dynamics it is necessary to know the forces acting on the vortices. Using a very general Berry phase arguments Ao and Thoules 23. concluded that Magnus force is the only transverse force on the vortex. If this is true, no transverse force from quasiparticles and impurities would act on the vortex. Recently Sonin 24,25] doubted this result arguing that the effective Magnus force, where Iordanskii force from quasiparticles [26] and Kopnin-Kravtsov force from impurities [27] are included should be taken into account. Krasnov and Logvenov [28] have used effective Magnus force proposed by Sonin for calculation of transport properties in the presence of transport current and temperature gradient. In the discussion of their results they omitted the Iordanskii force and moreover they did not take into account reaction force of vortices on superconducting and normal particles.

In our model we suppose that the Magnus force is responsible for the interaction between vortices and superconducting fluid, while Lorentz force (which is equivalent to the Iordanskii force) is responsible for the interaction between vortices and the normal state fluid. In our approach we treat the vortices, superconducting and normal state fluid as three mutually interacting subsystems 
and simultaneously solve their equations of motion. The solution quite naturally explains the Hall voltage sign reversal and simple numerical calculations showed that the model is able to explain at least qualitatively the experimental data. Moreover it is shown that Hall anomaly exists in the flux flow regime, i.e. it is not necessary to take into account the pinning.

\section{THE MODEL}

Vortices, superconducting and normal state fluids form three mutually interacting subsystems, consequently their equations of motion must be solved simultaneously. At the beginning let us briefly summarize the forces felt by the vortices (v), superconducting (s) and normal state (n) charge carriers. Vortices interact with superconducting charge carriers by the Magnus force. If a vortex oriented along the z-axis moves with velocity $\mathbf{v}_{L}$ then it feels the force

$$
\begin{aligned}
\mathbf{F}_{M}(v) & =\frac{e}{|e|} \frac{n_{s} h}{2}\left(\mathbf{v}_{s}-\mathbf{v}_{L}\right) \times \mathbf{z} \\
& =\frac{e}{|e|} m_{v} f_{s} \Omega\left(\mathbf{v}_{s}-\mathbf{v}_{L}\right) \times \mathbf{z}
\end{aligned}
$$

where $n_{s}=f_{s} n$ is the density of superconducting charge carriers having electric charge $e$ and velocity $\mathbf{v}_{s}$. Vortices are supposed to behave like quasiparticles; $m_{v}$ denotes vortex mass per unit length. If the superconducting fluid does not move the Magnus force is perpendicular to the vortex velocity so that in absence of damping the free vortex makes circular motion with angular frequency $\Omega=$ $n h / 2 m_{v}$. The superconducting charge carriers must feel the reaction force

$$
\mathbf{F}_{M}(s)=-\frac{n_{v}}{n_{s}} \mathbf{F}_{M}(v)=-\frac{e}{|e|} m \omega_{c}\left(\mathbf{v}_{s}-\mathbf{v}_{L}\right) \times \mathbf{z},
$$

where $n_{v}$ is the vortex density and $\omega_{c}=e B / m$ is the cyclotron frequency of superconducting charge carriers having the effective mass $m$ in the magnetic field $\mathbf{B}=n_{v} \Phi_{0} \mathbf{z}$, which is the averaged field inside the superconductor.

Let us consider the vortex system, in which the mean distance between vortices is small in comparison with the penetration depth. In this case the magnetic field in the superconductor is almost homogeneous and the normal state charge carriers moving with the velocity $\mathbf{v}_{n}$ must feel the Lorentz force. Using Aharonov-Casher lagrangian 29] it can be shown that also in this case relative velocity $\mathbf{v}_{n}-\mathbf{v}_{L}$ is decisive, so that the force felt by the superconducting charge carriers can be written in following form

$$
\mathbf{F}_{L}(n)=e\left(\mathbf{v}_{n}-\mathbf{v}_{L}\right) \times \mathbf{B}=\frac{e}{|e|} m \omega_{c}\left(\mathbf{v}_{n}-\mathbf{v}_{L}\right) \times \mathbf{z} .
$$

Vortices carrying the magnetic field act on the normal state fluid, and consequently they must feel the reaction force

$$
\begin{aligned}
\mathbf{F}_{L}(v)=-\frac{n_{n}}{n_{v}} \mathbf{F}_{L}(n) & =-\frac{e}{|e|} f_{n} \frac{n h}{2}\left(\mathbf{v}_{n}-\mathbf{v}_{L}\right) \times \mathbf{z} \\
& =-\frac{e}{|e|} m_{v} f_{n} \Omega\left(\mathbf{v}_{n}-\mathbf{v}_{L}\right) \times \mathbf{z}
\end{aligned}
$$

To simplify the formulae in the following text the electric charge $e$ is supposed to be positive. Vortex pinning and damping will be characterized by the vortex pinning frequency $\alpha=\sqrt{\kappa / m_{v}}$ ( $\kappa$ is the commonly used pinning constant) and vortex relaxation time $\tau_{v}$, respectively. The normal state fluid damping will be characterized by the normal state fluid relaxation time $\tau_{n}$. In the Hall effect experiments the electric current $\mathbf{j}=j_{x} \mathbf{x}$ is controlled experimentally and the velocities $\mathbf{v}_{L}, \mathbf{v}_{s}$, $\mathbf{v}_{n}$ together with the electric field $\mathbf{E}$ must be determined from the expression for current

$$
\mathbf{j}=n e\left(f_{s} \mathbf{v}_{s}+f_{n} \mathbf{v}_{n}\right)
$$

and from the following three equations of motion

$$
\begin{gathered}
\dot{\mathbf{v}}_{s}=\frac{e}{m} \mathbf{E}-\omega_{c}\left(\mathbf{v}_{s}-\mathbf{v}_{L}\right) \times \mathbf{z} \\
\dot{\mathbf{v}}_{n}=\frac{e}{m} \mathbf{E}+\omega_{c}\left(\mathbf{v}_{s}-\mathbf{v}_{L}\right) \times \mathbf{z}-\frac{1}{\tau_{n}} \mathbf{v}_{n} \\
\dot{\mathbf{v}}_{L}=-\alpha^{2} \mathbf{r}_{L}-\frac{1}{\tau_{v}} \mathbf{v}_{L}+f_{s} \Omega\left(\mathbf{v}_{s}-\mathbf{v}_{L}\right) \times \mathbf{z} \\
-f_{n} \Omega\left(\mathbf{v}_{n}-\mathbf{v}_{L}\right) \times \mathbf{z}
\end{gathered}
$$

The same set of equations has been recently used successfully for the study of high frequency vortex dynamics and for interpretation of the far infrared magnetoconductivity 30 .

In the steady state the accelerations on the left hand sides of the equations of motion are equal to zero. The solution depends on the magnitude of the current density used for the measurement. For high currents Magnus force exceeds the vortex pinning, vortices move and in the first approximation the effect of vortex pinning can be neglected. After substituting $\alpha=0$ to equation of motion (6) the vortex velocity is

$$
\begin{aligned}
& v_{L x}=\left[\left(\tau_{n}^{2} \omega_{c}^{2}+f_{s}^{2}\left(f_{s}-f_{n}\right)\right) \tau_{v} \Omega+2 \tau_{n} \omega_{c} f_{n} f_{s}\right] \beta j_{x} \\
& v_{L y}=-\left[2 \tau_{n} \omega_{c} \tau_{v} \Omega f_{n} f_{s}+\tau_{n}^{2} \omega_{c}^{2}\left(f_{s}-f_{n}\right)-f_{s}^{2}\right] \beta j_{x}
\end{aligned}
$$

where $\beta=\tau_{v} \Omega /$ Dne and

$$
\begin{aligned}
D & =\left[\tau_{v}^{2} \Omega^{2}+\left(f_{s}-f_{n}\right)^{2}\right] \tau_{n}^{2} \omega_{c}^{2}+8 f_{s}^{2} f_{n} \tau_{n} \omega_{c} \tau_{v} \Omega \\
& +f_{s}^{2}\left(f_{s}-f_{n}\right)^{2} \tau_{v}^{2} \Omega^{2}+f_{s}^{2}
\end{aligned}
$$

The total current in the $\mathrm{x}$-axis direction $j_{x}$ is the sum of the superconducting current

$$
\begin{aligned}
j_{s x} & =\left[\left(f_{s}\left(f_{s}-f_{n}\right)^{2}+\tau_{n}^{2} \omega_{c}^{2}\right) \tau_{v}^{2} \Omega^{2}+6 f_{n} f_{s} \tau_{n} \omega_{c} \tau_{v} \Omega\right. \\
& \left.+\tau_{n}^{2} \omega_{c}^{2}\left(f_{s}-f_{n}\right)+f_{s}\right] \frac{f_{s}}{D} j_{x}
\end{aligned}
$$


and the normal state current

$$
\begin{array}{r}
j_{n x}=\left[\tau_{n}^{2} \omega_{c}^{2} \tau_{v}^{2} \Omega^{2}+2 f_{s}^{2} \tau_{n} \omega_{c} \tau_{v} \Omega\right. \\
\left.-\tau_{n}^{2} \omega_{c}^{2}\left(f_{s}-f_{n}\right)\right] \frac{f_{n}}{D} j_{x}
\end{array}
$$

while the superconducting current in the y-axis direction is cancelled by the normal fluid backflow current

$$
\begin{aligned}
j_{s y}=- & j_{n y}=\left[-\tau_{n} \omega_{c} \tau_{v}^{2} \Omega^{2}\left(f_{s}-f_{n}\right)\right. \\
& \left.+2 \tau_{n}^{2} \omega_{c}^{2} \tau_{v} \Omega+\tau_{n} \omega_{c}\right] \frac{f_{n} f_{s}}{D} j_{x},
\end{aligned}
$$

The longitudinal and Hall resistivities may be expressed as

$$
\begin{aligned}
\rho_{x x} & =\frac{E_{x}}{j_{x}}=\left[\tau_{n} \omega_{c} f_{n}\left(1+\tau_{v}^{2} \Omega^{2}\right)\right. \\
& \left.+\tau_{v} \Omega\left(f_{s}^{2}+\tau_{n}^{2} \omega_{c}^{2}\right)\right] \frac{\omega_{c}}{\epsilon_{0} \omega_{p}^{2} D} \\
\rho_{x y} & =\frac{E_{y}}{j_{x}}=\left[\left(f_{s}-f_{n}\right)\left(f_{s} f_{n} \tau_{v}^{2} \Omega^{2}-\tau_{n}^{2} \omega_{c}^{2}\right)\right. \\
& \left.-f_{s}\left(1+4 f_{n} \tau_{n} \omega_{c} \tau_{v} \Omega\right)\right] \frac{\omega_{c}}{\epsilon_{0} \omega_{p}^{2} D} .
\end{aligned}
$$

The last formula quite naturally explains the change of sign in the Hall effect measurements. At zero temperature where $f_{n}=0$, the Hall resistivity $\rho_{x y}=$ $-\omega_{c} / \epsilon_{0} \omega_{p}^{2}\left(1+\tau_{v}^{2} \Omega^{2}\right)$ is negative, while for temperatures above $T_{c}$ it has the positive value $\rho_{x x}=\omega_{c} / \epsilon_{0} \omega_{p}^{2}$. The conductivity may be expressed as

$$
\begin{aligned}
\sigma_{x x} & =\frac{\epsilon_{0} \omega_{p}^{2}}{\omega_{c} \gamma}\left[f_{n} \tau_{n} \omega_{c}\left(1+\tau_{v}^{2} \Omega^{2}\right)+\tau_{v} \Omega\left(f_{s}^{2}+\tau_{n}^{2} \omega_{c}^{2}\right)\right], \\
\sigma_{x y} & =\frac{\epsilon_{0} \omega_{p}^{2}}{\omega_{c} \gamma}\left[\left(f_{s}-f_{n}\right)\left(\tau_{n}^{2} \omega_{c}^{2}-f_{s} f_{n} \tau_{v}^{2} \Omega^{2}\right)\right. \\
& \left.+4 f_{s} f_{n} \tau_{n} \omega_{c} \tau_{v} \Omega+f_{s}\right]
\end{aligned}
$$

where $\gamma=1+\left(\tau_{n} \omega_{c}+f_{n} \tau_{v} \Omega\right)^{2}$. It is interesting to note, that using $\omega_{c}=e B / m$ for low magnetic field it is possible to approximate the Hall conductivity as

$$
\sigma_{x y}=A_{-1} / B+A_{0}+A_{1} B .
$$

In the low temperature region, where the dependence of normal state fraction $f_{n}$ on the magnetic field is negligible the coefficients can be written as

$$
\begin{aligned}
& A_{-1}=\epsilon_{0} \omega_{p}^{2} \frac{m}{e} \frac{\left.f_{s}^{2} \tau_{v} \Omega\right)}{1+f_{n}^{2} \tau_{v}^{2} \Omega^{2}} \\
& A_{0}=\epsilon_{0} \omega_{p}^{2} f_{n} \tau_{n} \frac{\left(1+f_{n}^{2} \tau_{v}^{2} \Omega^{2}\right)^{2}-f_{s}^{2} \tau_{v}^{2} \Omega^{2}}{\left(1+f_{n}^{2} \tau_{v}^{2} \Omega^{2}\right)^{2}} \\
& \left.A_{1}=\epsilon_{0} \omega_{p}^{2} \frac{e}{m} \tau_{v} \Omega\right) \tau_{v} \Omega \tau_{n}^{2} \\
& \times \frac{\left[\left(1+f_{n}^{2} \tau_{v}^{2} \Omega^{2}\right)^{2}-f_{n}^{2} f_{s}\left(f_{s}+4\right) \tau_{v}^{2} \Omega^{2}-f_{s}\left(1+3 f_{n}\right)\right]}{\left(1+f_{n}^{2} \tau_{v}^{2} \Omega^{2}\right)^{3}} .
\end{aligned}
$$

\section{RESULTS}

The above presented formulae should be applicable mainly to high temperature superconductors, because in these materials the London penetration length is rather large,

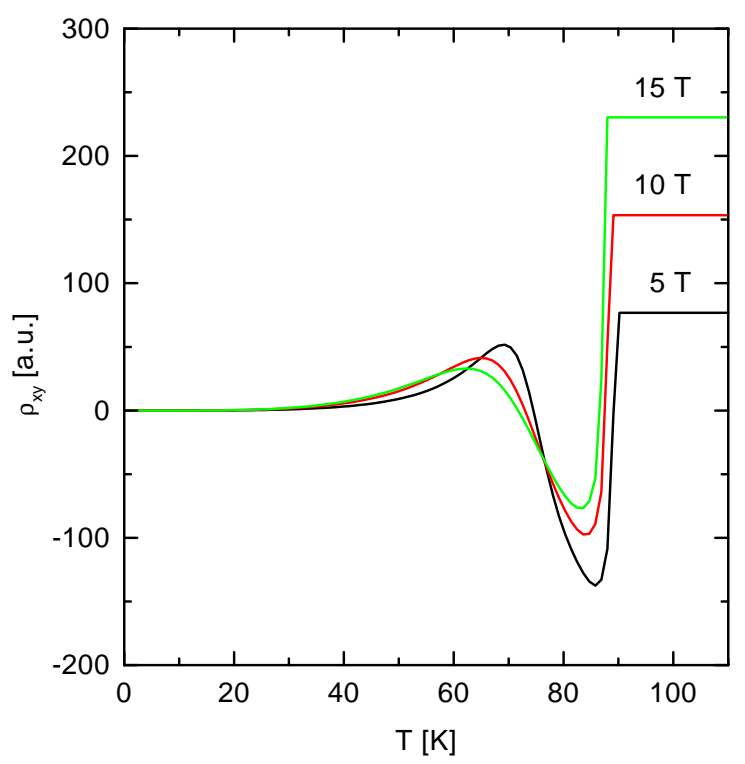

FIG. 1. Temperature dependence of the Hall resistivity for different magnetic fields. Parameters of the model are $\Omega_{0} \tau_{v}=50, \alpha=0,1 / \tau_{n}=35 \mathrm{~cm}^{-1}$.

so that even in moderate fields the magnetic field in the superconductors is almost homogeneous. The coherence length is small, so that the redistribution of charge and current density in the normal state core may be neglected. To determine the theoretical dependence of the Hall resistance on the temperature and magnetic field, it is necessary to estimate the dependence of the parameters involved. We suppose that $f_{n}=\left(T / T_{c}\right)^{4}$ where the critical temperature $T_{c}$ depends on the magnetic field as $T_{c}=T_{c 0} \sqrt{1-B / B_{c 2}}$. Here $T_{c 0}$ and $B_{c 2}$ are the critical temperature at zero magnetic field and critical magnetic field at zero temperature, respectively. Using the Hsu's expression for the vortex mass $m_{v}=\left(\pi^{2} / 4\right) m k_{F}^{2} \xi^{2}$ [31], the expression for the Fermi wave vector $k_{F}^{2}=2 \pi n$ and $\xi=\hbar v_{F} / \pi \Delta$ for the coherence length, it is possible to show that $\Omega=\Delta^{2} / E_{F}$. Supposing that the Fermi energy $E_{F}$ is a constant, while the gap depends on the temperature and magnetic field approximately as $\Delta=\Delta_{0} \sqrt{\cos \left(\left(\pi T^{2} / 2 T_{c}^{2}\right)\right.}$ [32], we will take $\Omega=\Omega_{0} \cos \left(\pi T^{2} / 2 T_{c}^{2}\right)$. The cyclotron frequency is proportional to the magnetic field $\omega_{c}=e B / m$, and the relaxation times $\tau_{n}, \tau_{v}$ are supposed to be constant in the first approximation. 


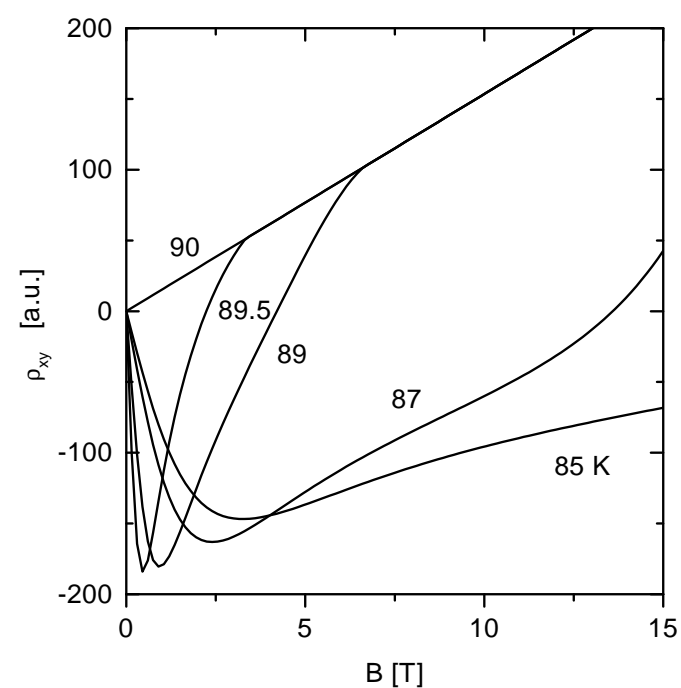

FIG. 2. Magnetic field dependence of the Hall resistivity for different temperatures. The parameters are same as in the Fig.1

Using above mentioned expressions the theoretical temperature and magnetic field dependencies of the Hall resistivity in the zero pinning regime (eq. 16) plotted in the Fig. 1, 2 reproduce qualitatively the data observed experimentally by many authors. The second sign change from negative to positive value is clearly seen from the Fig.1 . Such behavior is observed experimentally in Bi , $\mathrm{Tl}$ and $\mathrm{Hg}$ based superconductors. Moreover detailed analysis of formula (16) reveals a third sign change at even lower temperatures. Such change of sign was recently observed by Kang et al. 33 on $\mathrm{HgBaCaCuO}$. However we should note that it is questionable if such experimentally observed third sign change is not a consequence of the presence of another superconducting phase as it was suggested in 34.

\section{CONCLUSION}

We have explained the Hall voltage sign reversal in the superconductors below the critical temperature considering vortices, superconducting and normal state fluids as mutually interacting subsystems where also reaction forces are taken into account. Results of calculations reveal up to three changes of sign of Hall resistivity with decreasing temperature. The experimentally observed dependence of the Hall conductivity on magnetic field [35,36] also confirms our results (eq. 19), where a term independent on magnetic field is added in contrast to the previous theories [37,38]. Without any special assumption about pinning strength or fluctuations as was made by Wang et al [39] the theoretical curves qualitatively explain experimental results of the Hall effect of super- conductors in the mixed state. It is worth noting that in the above presented theory the boundary conditions on the sample surface are not considered. It seems clear that for quantitative description of the experimental data finite dimensions of the measured sample should be taken into account.

\section{ACKNOWLEDGMENTS}

This work was supported by MŠMT project Kontakt ME 160 and GACR $\sharp 106 / 99 / 1441$, GACR $\sharp 104 / 99 / 1440$, GAAV A1010919 grants .

[1] J. Bardeen and M. J. Stephen, Phys. Rev. B 49, 1197 (1965)

[2] P. Nozieres and W. F. Vinnen, Phil. Mag. 14, 667 (1966)

[3] S. J. Hagen, C. J. Lobb, R. L. Green, M. G.Forrester and J. Talvacchio, Phys. Rev. B 42, 6777 (1990)

[4] H.Fukuyama, H.Ebisawa, T.Tsuzuki Prog.Theor.Phys. 46, 1028 (1971)

[5] T.Nishio, H.Ebisawa, Physica C 290, 43 (1997)

[6] H.J. Jensen, P. Minnhagen, E. Sonin and H. Weber, Europhysics Lett. 20, 463 (1992)

[7] M. Viret and J. M. D. Coey, Phys. Rev. B 49, 3457 (1994)

[8] P. Ao, J.Phys.Condens.Matter 10, L667 (1998)

[9] Z.D.Wang, J. Dong and C.S. Ting, Phys. Rev. Lett. 72, 3875 (1994)

[10] B. Y. Zhu, D. Y. Xing, Z. D. Wang, B. R. Zhao and Z. X. Zhao, Phys. Rev. B 60, 3080 (1999)

[11] N. B. Kopnin and V. M. Vinokur, Phys.Rev.Lett. 83, 4864, (1999)

[12] D.A.Beam, N.-C.Yeh, F.Holtzberg, J.Phys.: Condens. Matter 10, 5955 (1998)

[13] R. C. Budhani, S. H. Liou and Z.X. Cai, Phys. Rev. Lett. 71, 621 (1993)

[14] W. Leibich, P. Gehringer, W. Göb, W. Lang, K. Bierleutgeb and D. Bäuerle, Physica C 282-287, 2321 (1997)

[15] W. Göb, W. Lang, J. D. Pedarning, R. Rössler and D. Bäuerle, Physica C317-318, 627 (1999)

[16] M. V. Feigelman , V.B. Geshkeibein, A. V. Larkin and V. M. Vinokur, JETP Lett 62, 834 (1995)

[17] D. I. Khomski and A. Freimuth, Phys. Rev. Lett. 75, 1384 (1995)

[18] Y. Matsuda, K.Kumagai , Proc. of LT22, Finland, 398 (1999)

[19] J.I.Martin, M.Velez, F.Guinea, J.L.Vicent, Phys.Rev. B 55,5659 (1997)

[20] K.Nakao, K.Hayash, T.Utagawa, Y.Enomoto, N.Koshizuka, Phys.Rev.B 57, 8662 (1998)

[21] Wang Yun-Ping, Zhang Dian-Lin, Zhao Bai-Ru, Modern Phys.Lett.B 12, 719 (1998)

[22] H.L.Ji, K.W.Wong, Phys.Lett.A 256, 66 (1999) 
[23] P. Ao and D. J. Thouless, Phys. Rev. Lett. 70, 2158 (1993)

[24] E. B. Sonin, Czech. J. Phys. 46, 911 (1996)

[25] E. B. Sonin, Phys. Rev. B 55, 485 (1997)

[26] S. V. Iordanskii, Sov. Phys. JETP 22, 160 (1966)

[27] N. B. Kopnin and V. E. Kravtsov, Sov. Phys. JETP 44, $861(1976)$

[28] V. M. Krasnov and G. Yu. Logvenov, Physica C 274, 268 (1997)

[29] Y. Aharonov and A. Casher, Phys. Rev. Lett. 53, 319 (1984)

[30] J. Koláček and E. Kawate, Phys. Lett. A 260, 300 (1999)

[31] T. C. Hsu, Physica C 213, 305 (1993)

[32] R. Pöpel, J. Appl. Phys. 66, 5950 (1989)

[33] W. N. Kang, B.W. Kang, Q.Y. Chen,J.Z. Wu, Y. Bai, W. K. Chu, D. K. Christen , R. Kerchner and S.-I. Lee, Phys.Rev.B 61, 722 (2000)

[34] P. Vašek, I. Janeček, Int. J. Mod. Phys. B 13, 3741 (1999)

[35] D. M. Ginsberg and J. T. Manson, Phys. Rev. B 51, 515 (1995)

[36] W.-S. Kim, W.N. Kang, S. J. Oh, M.-S. Kim, Y. Bai, S.-I. Lee, CH. H. Choi and H.-C. Ri, Physica C324, 77 (1999)

[37] A. T. Dorsey, Phys. Rev B46, 8376 (1992)

[38] N. B. Kopnin, B. I. Ivlev, V. A. Kalatsky, J. Low. Temp. Phys. 90,1 (1993)

[39] Z. D. Wang, J. Dong and C. S. Ting, Phys. Rev. Lett. 72, 3875 (1994) 